\title{
The Role of Bcc Mg/Nb Interfaces in Nanocomposite Deformation Observed via In- Situ Mechanical Testing in TEM
}

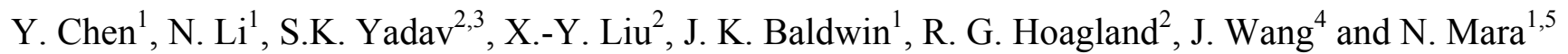 \\ 1. MPA-CINT, Los Alamos National Laboratory, Los Alamos, NM, USA \\ 2. MST-8, Los Alamos National Laboratory, Los Alamos, NM, USA \\ 3. Department of Metallurgical and Materials Engineering, Indian Institute of Technology (IIT) Madras, \\ Chennai, India. \\ 4. Mechanical and Materials Engineering, University of Nebraska-Lincoln, Lincoln, NE \\ , USA \\ 5. Institute for Materials Science, Los Alamos National Laboratory, Los Alamos, NM, USA
}

Magnesium ( $\mathrm{Mg}$ ) alloys attract intense attention for being one of most promising lightweight structural materials for automobile and aerospace applications [1,2]. However, their low strength and poor ductility, caused by scarcity of easy slip systems and localized shear due to twinning in hexagonal closepacked (hcp) structures limit their applicability. [3,4]. Improving the strength without sacrificing ductility remains a significant challenge. Many techniques, such as non-traditional processing $[5,6]$, grain refinement [7], and alloying with rare earth elements [8], have been applied to tune the relative activity of slip and twinning in $\mathrm{Mg}$ alloys, improving deformability while maintaining strength. $\mathrm{Mg}$ and $\mathrm{Mg}$ alloys can also be strengthened through tailoring the microstructure of $\mathrm{Mg}$-based composites, such as in $\mathrm{Mg}$ metal laminates [9-11].

$\mathrm{Mg} / \mathrm{Nb}$ nanolaminates have been proposed to be effective in improving ductility and strength in $\mathrm{Mg}$. Since bcc $\mathrm{Mg}$ can be stabilized by being sandwiched in between bcc/bcc $\mathrm{Mg} / \mathrm{Nb}$ interfaces when the individual layer thickness is below $5 \mathrm{~nm}[9,10]$, the ductility is improved as bec $\mathrm{Mg}$ has additional active room temperature slip systems as compared to hcp $\mathrm{Mg}$. However, the experimental evidence supporting this is still sparse. In situ mechanical testing is a useful tool for real-time observation of deformation mechanisms at nanometer scales inside a transmission electron microscope (TEM). Our results directly validate the hypothesis that bcc $\mathrm{Mg}$ could accommodate severe plastic deformation. Moreover, in situ high-resolution TEM (HRTEM) reveals that in bcc/bcc $\mathrm{Mg} / \mathrm{Nb}$, a reversible bcc-hcp phase transformation in $\mathrm{Mg}$ could occur during loading and unloading, as shown in figure 1.

In summary, the fundamental understanding of deformation mechanisms of bec $\mathrm{Mg}$ in $\mathrm{Mg} / \mathrm{Nb}$ laminates is investigated. The bcc $\mathrm{Mg}$ exhibits enhanced ductility due to (1) additional active slip systems in comparison to hep $\mathrm{Mg}$ and (2) a bcc-hcp phase transformation in Mg layers. The enhanced ductility exhibited by nanoarchitectured $\mathrm{Mg}$ in this investigation may have future implications in the design of Mg alloys [12].

\section{References:}

[1] Froes, F., Materials Science and Engineering: A, 184 (1994), p. 119.

[2] Mordike, B. and T. Ebert, Materials Science and Engineering: A, 302 (2001), p. 37.

[3] Christian, J.W. and S. Mahajan, Progress in materials science, 39 (1995), p. 1.

[4] Partridge, P., Metallurgical reviews, 12 (1967), p. 169.

[5] Chino, Y. et al, Materials Science and Engineering: A, 441 (2006), p. 349. 
[6] Kim, S.-H. et al, Materials Letters, 59 (2005), p. 3876.

[7] Barnett, M.R. et al, Acta Materialia, 52 (2004), p. 5093.

[8] Tekumalla, S. et al, Metals, 5 (2014), p. 1.

[9] Ham, B. and X. Zhang, Materials Science and Engineering: A, 528 (2011), p. 2028.

[10] Kumar, A., I.J. Beyerlein, and J. Wang, Applied Physics Letters, 105 (2014), p. 071602.

[11] Lu, Y. et al, Acta Materialia, 63 (2014), p. 216.

[12] The authors acknowledge the Center for Integrated Nanotechnologies, an Office of Science User Facility operated for the U.S. Department of Energy (DOE) Office of Science. Los Alamos National Laboratory, an affirmative action equal opportunity employer, is operated by Los Alamos National Security, LLC, for the National Nuclear Security Administration of the U.S. Department of Energy under contract DE-AC52-06NA25396. This work was partly supported by the U.S. Department of Energy, Office of Science, Office of Basic Energy Sciences. J.W. acknowledges the support by the Nebraska Center for Energy Sciences Research (NCESR) at the University of Nebraska-Lincoln (UNL).

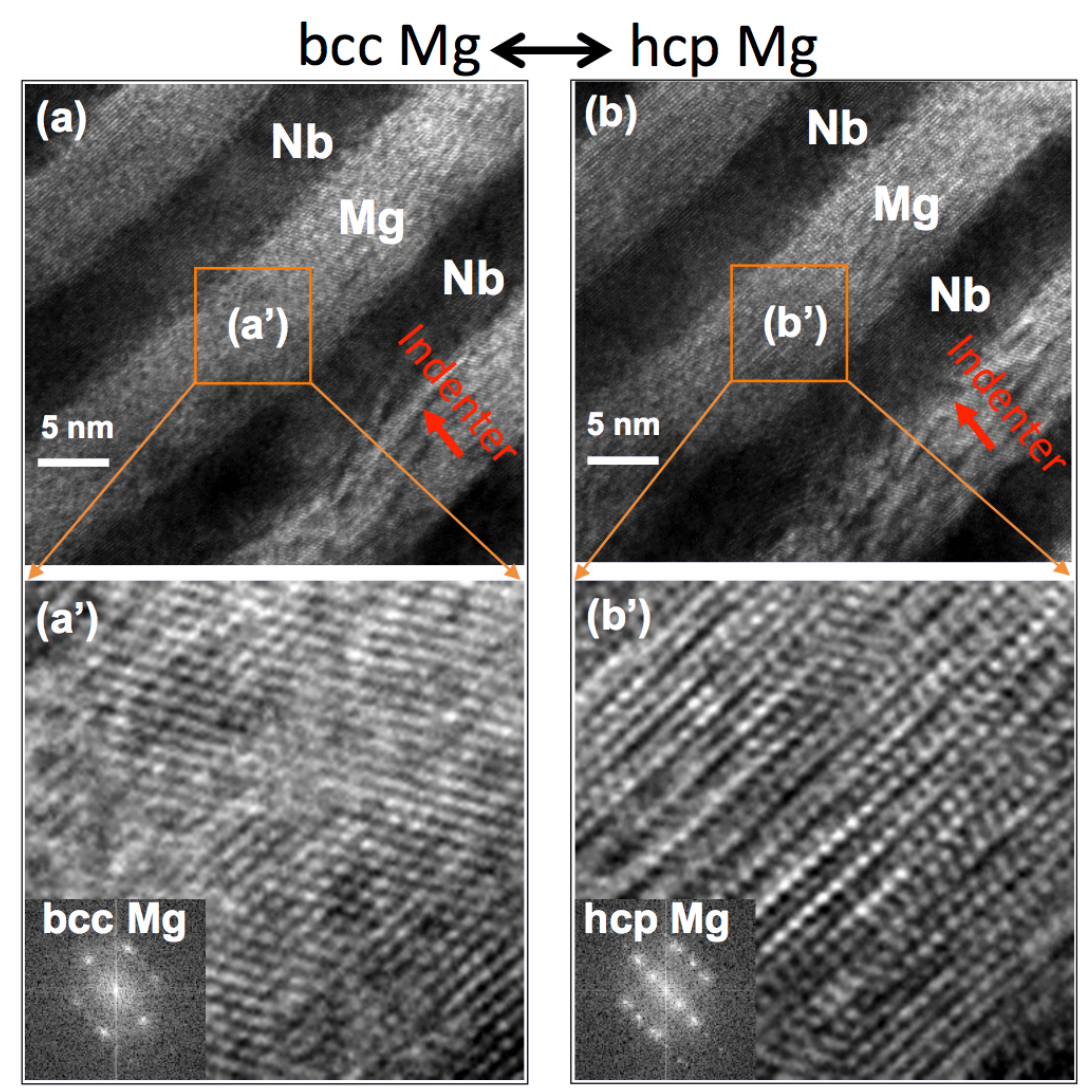

Figure 1. The phase transformation of $\mathrm{Mg}$ from bcc $\mathrm{Mg}$ to hcp Mg during in situ nanoindentation inside a transmission electron microscope. (a) HRTEM snapshot and (a') magnified image demonstrate bcc structure of Mg by the inset FFT (a'); (b) HRTEM snapshot and (b') magnified image demonstrate hcp structure of Mg by the inset FFT. (a) and (b) are from same location. 